\title{
Graphene oxide 2D films for integrated waveguide and ring resonator polarizers
}

\author{
Jiayang $\mathrm{Wu}^{\mathrm{a}}$, Yunyi Yang ${ }^{\mathrm{a}}$, Yuning Zhang ${ }^{\mathrm{a}}$, Yang $\mathrm{Qu}^{\mathrm{a}}$, Xingyuan $\mathrm{Xu}^{\mathrm{a}}$, Linnan Jia ${ }^{\mathrm{a}}$, Yao Liang ${ }^{\mathrm{a}}$, \\ Sai T. Chu ${ }^{\text {b }}$, Brent E. Little ${ }^{c}$, Roberto Morandotti ${ }^{\text {d, e, f }}$, Baohua Jia ${ }^{\text {a }}$, and David Moss ${ }^{\text {a, * }}$ \\ ${ }^{a}$ Centre for Micro-Photonics, Swinburne University of Technology, Hawthorn, VIC 3122, Australia \\ ${ }^{\mathrm{b}}$ City University of Hong Kong, Tat Chee Avenue, Hong Kong, China \\ 'Xi'an Institute of Optics and Precision Mechanics Precision Mechanics of CAS, Xi'an, China

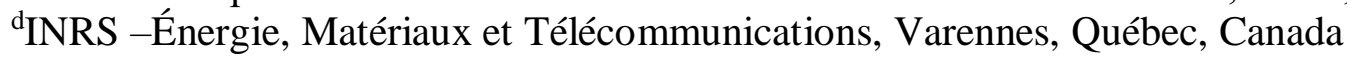 \\ ${ }^{\text {e }}$ ITMO University, St. Petersburg, Russia \\ fUniversity of Electronic Science and Technology of China, Chengdu, China \\ * Electronic mail: dmoss@swin.edu.au
}

\begin{abstract}
Polarization selective devices, such as polarizers and polarization selective resonant cavities (e.g., gratings and ring resonators), are core components for polarization control in optical systems and find wide applications in polarizationdivision-multiplexing, coherent optical detection, photography, liquid crystal display, and optical sensing. In this paper, we demonstrate integrated waveguide polarizers and polarization-selective micro-ring resonators (MRRs) incorporated with graphene oxide (GO). We achieve highly precise control of the placement, thickness, and length of the GO films coated on integrated photonic devices by using a solution-based, transfer-free, and layer-by-layer GO coating method followed by photolithography and lift-off processes. The latter overcomes the layer transfer fabrication limitations of 2D materials and represent a significant advance towards manufacturing integrated photonic devices incorporated with 2D materials. We measure the performance of the waveguide polarizer for different GO film thicknesses and lengths versus polarization, wavelength, and power, achieving a very high polarization dependent loss (PDL) of $\sim 53.8 \mathrm{~dB}$. For GOcoated integrated MRRs, we achieve an 8.3-dB polarization extinction ratio between the TE and TM resonances, with the extracted propagation loss showing good agreement with the waveguide results. Furthermore, we present layer-by-layer characterization of the linear optical properties of 2D layered GO films, including detailed measurements that conclusively determine the material loss anisotropy of the GO films as well as the relative contribution of film loss anisotropy versus polarization-dependent mode overlap, to the device performance. These results offer interesting physical insights and trends of the layered GO films from monolayer to quasi bulk like behavior and confirm the high-performance of integrated polarization selective devices incorporated with GO films.
\end{abstract}

Keywords: 2D materials, integrated photonics, material anisotropy, polarization control, graphene oxide

\section{INTRODUCTION}

Polarization control is a fundamental requirement in many optical technologies [1-5]. Integrated polarization-selective devices based on complementary metal-oxide-semiconductor (CMOS) compatible integrated platforms $[1,6,7]$, offering advantages of compact footprint, high stability, mass producibility and high scalability [8-12], are functional building blocks for photonic integrated circuits. In recent years, the huge optical anisotropy and broadband response of twodimensional (2D) materials such as graphene and transition metal dichalcogenides have been widely recognized and exploited to implement polarization-selective devices [13-16]. Generally, the integration of 2D materials on CMOS compatible platforms requires layer transfer processes [16-18], where exfoliated or chemical vapour deposition grown 2D membranes are attached onto dielectric substrates (e.g., silicon and silica wafers). Despite its widespread implementation, the transfer approach itself is complex, which makes it difficult to achieve precise patterning, as well as flexible placement and large-area continuous coating on integrated devices. Accurate control of the layer position, thickness and size is critical for optimizing parameters such as mode overlap and loss for performance. Current methods significantly limit the scale of fabrication for integrated devices incorporating 2D materials. 
Owing to its ease of preparation as well as the tunability of its material properties, graphene oxide (GO) has become a highly promising member of the 2D family [19-22]. In Ref. [15], a broadband GO-polymer waveguide polarizer with a high PDL of $\sim 40 \mathrm{~dB}$ was demonstrated, where the GO films were introduced onto an SU8 polymer waveguide using dropcasting methods. The GO film thickness for each drop-casting step was $\sim 0.5 \mu \mathrm{m}$ and the drop coating diameter was $\sim 1.3$ $\mathrm{mm}$, neither being ideal for achieving precise control of the placement, thickness, and length of the GO films.

Recently [19], we reported large-area, transfer-free, and high-quality GO film coating on integrated waveguides using a solution-based method with layer-by-layer deposition of GO films. Here, we use these techniques to demonstrate GOcoated integrated waveguide polarizers and polarization-selective micro-ring resonators (MRRs) on a CMOS compatible doped silica platform [20]. We achieve highly precise control of the placement, thickness, and length of the GO films coated on integrated photonic devices by using our layer-by-layer GO coating method followed by photolithography and lift-off processes. We measure the performance of the waveguide polarizer for different GO film thicknesses and lengths versus polarization, wavelength, and power, achieving a very high polarization dependent loss (PDL) of $\sim 53.8 \mathrm{~dB}$. For GO-coated MRRs, we achieve an 8.3-dB polarization extinction ratio between the TE and TM resonances. These results confirm the high-performance of integrated polarization selective devices incorporated with 2D layered GO films.

\section{GO-COATED WAVEGUIDE POLARIZERS}

Figure 1(a) shows a schematic of a uniformly GO-coated waveguide polarizer. The waveguides were fabricated from highindex doped silica glass core surrounded by silica via CMOS compatible processes [23-25] with chemical mechanical polishing (CMP) used as the last step to remove the upper cladding, so as to enable GO film coating on the top surface of the waveguide. GO coating was achieved with a solution-based method that yielded layer-by-layer GO film deposition, as reported previously [19]. Four steps for in-situ assembly of monolayer GO films were repeated to construct multilayer GO films on the desired substrate, with the process being highly scalable.
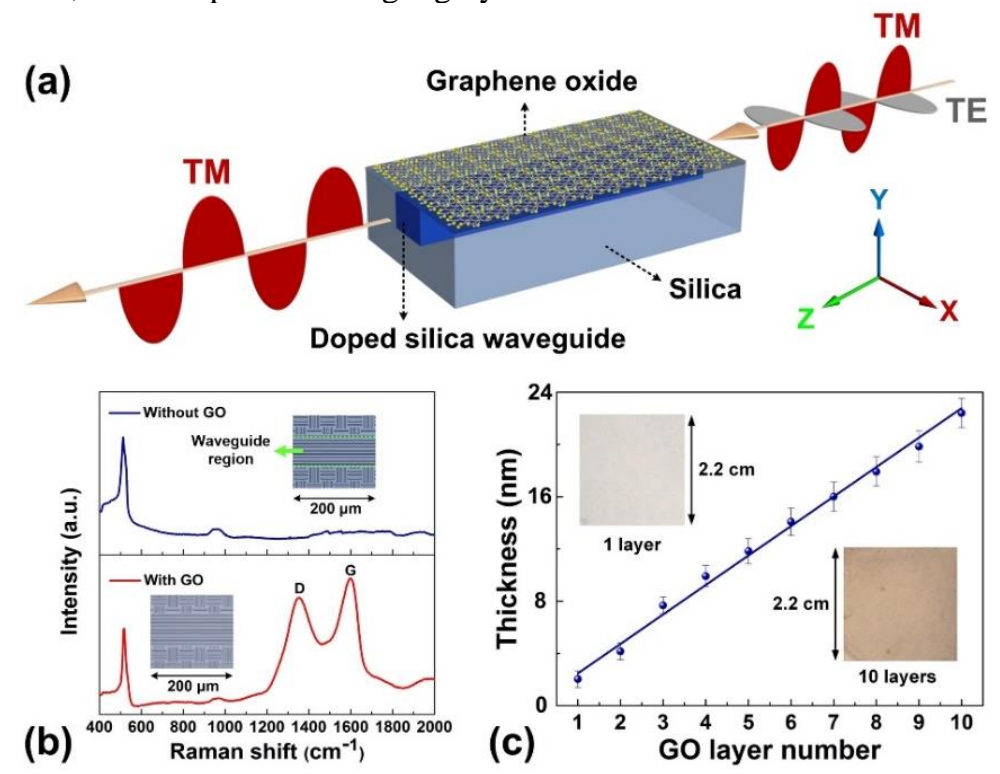

Figure 1. (a) Schematic illustration of GO-coated integrated waveguide polarizer. (b) Raman spectra of the integrated chip without GO and with 2 layers of GO. Insets show the corresponding microscope images with 9 parallel waveguides in the guiding region. (c) Measured GO film thickness versus GO layer number. Insets show the images of a $2.2 \mathrm{~cm} \times 2.2 \mathrm{~cm}$ silica substrate coated with 1 and 10 layers of GO, respectively.

We uniformly coated waveguides with 1 to 10 layers of GO. Figure 1(b) shows the measured Raman spectra of the waveguides without GO and with 2 layers of GO, confirming the integration of GO onto the top surface by the presence of the D (1345 cm-1) and G (1590 cm-1) peaks of GO. The microscope images of the integrated waveguide with zero and 2 layers of GO are shown in the insets, which illustrate the good morphology of the GO films. Figure 1(c) shows the thickness of GO films versus the layer number characterized by atomic force microscopy. The insets show images of 1 
and 10 layers of GO coated on a $2.2 \mathrm{~cm} \times 2.2 \mathrm{~cm}$ silica substrate with high uniformity. The dependence of GO film thickness versus layer number shows a nearly linear relationship, with a thickness of $\sim 2.18 \mathrm{~nm}$ on average for each layer.

In addition to the uniformly coated devices, we selectively patterned areas of GO films using lithography and lift-off processes. Apart from allowing precise control of the size and placement of the GO films, this enabled us to test the performance of the GO-coated waveguide polarizers with shorter GO coating lengths but higher film thicknesses (up to 100 layers). The chip was first spin-coated with photoresist and then patterned using photolithography to open a window on the waveguide. Next, GO films were coated on the chip using the method mentioned above and patterned via a lift-off process.

We used an 8-channel single-mode fiber (SMF) array to butt couple both TE and TM polarized continuous-wave (CW) light from a tunable laser near $1550 \mathrm{~nm}$ into the waveguides. The mode coupling loss between the SMF array and the waveguides was $\sim 8 \mathrm{~dB} /$ facet, which can readily be reduced to $\sim 1.0 \mathrm{~dB} /$ facet with mode convertors $[26,27]$. The propagation loss of the uncoated 1.5 -cm-long waveguides was very low $(<0.25 \mathrm{~dB} / \mathrm{cm})$ and so the total insertion loss $(\mathrm{TE}=-16.2 \mathrm{~dB} ; \mathrm{TM}=-16.5 \mathrm{~dB})$ of the uncoated devices was dominated by mode coupling loss. The slight PDL of the uncoated waveguides resulted mainly from a slightly different mode-coupling mismatch but possibly also polarization dependent scattering loss from the roughness of the polished top surface.

To characterize the performance of the devices, we introduce two figures of merit (FOMs) - one for the excess propagation loss $\left(\mathrm{FOM}_{\mathrm{EPL}}\right)$ and one for the overall polarization dependent loss $\left(\mathrm{FOM}_{\mathrm{PDL}}\right)$ :

$$
\begin{gathered}
\mathrm{FOM}_{\mathrm{EPL}}=\left(\mathrm{EPL}_{\mathrm{TE}}-\mathrm{EPL}_{\mathrm{TM}}\right) / \mathrm{EPL}_{\mathrm{TM}}, \\
\mathrm{FOM}_{\mathrm{PDL}}=\mathrm{PDL} / \mathrm{EIL},
\end{gathered}
$$

where the excess propagation losses, $\mathrm{EPL}_{\mathrm{TE}}(\mathrm{dB} / \mathrm{cm})$ and $\mathrm{EPL}_{\mathrm{TM}}(\mathrm{dB} / \mathrm{cm})$, are GO-induced excess propagation losses for the TE and TM polarizations, respectively. PDL $(\mathrm{dB})$ is the polarization dependent loss defined as the ratio of the maximum to minimum insertion losses. The excess insertion loss, EIL (dB), is the insertion loss induced by the GO film over the uncoated waveguide. The EIL only considers the insertion loss induced by GO, while excluding from the overall insertion loss both the mode coupling loss between the SMF array and the waveguide as well as the propagation loss of the uncoated waveguide. In our case, since the TM polarization had the lowest insertion loss, EIL is the excess GO-induced insertion loss for the TM polarization and is given by $\mathrm{EIL}=\mathrm{EPL}_{\mathrm{TM}} \cdot \mathrm{L}$, where $\mathrm{L}$ is the $\mathrm{GO}$ coating length. Note that $\mathrm{FOM}_{\mathrm{EPL}}$ only considers the propagation loss difference induced by the GO films, and so is more accurate for the characterization of their material anisotropy, whereas $\mathrm{FOM}_{\mathrm{PDL}}$ is more widely used for evaluating the device performance [16] since it also includes the background (uncoated) PDL. FOM EPL $_{\text {equals FOM }}$ PDL only when the TE and TM polarized insertion losses of the uncoated waveguide are the same.
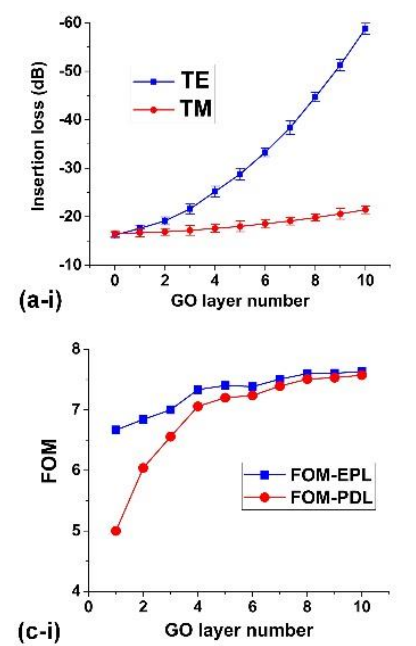
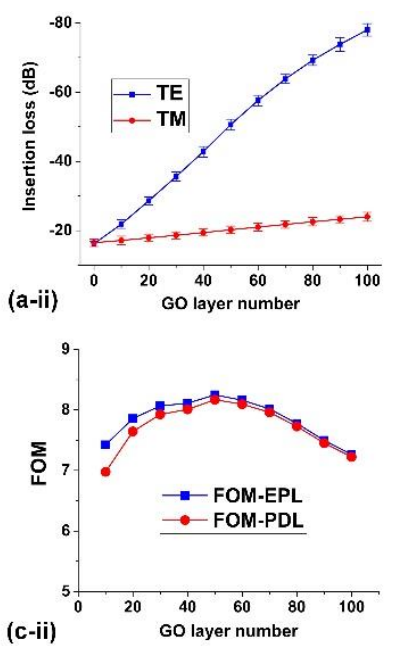
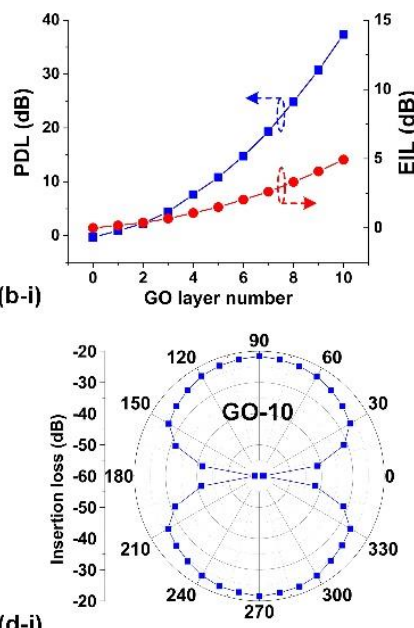
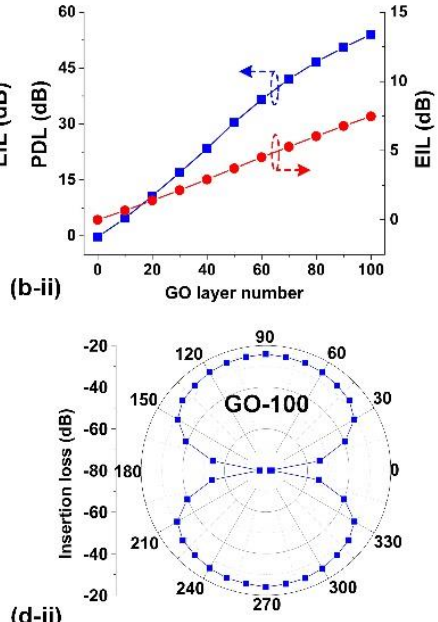

Figure 2. (a) Measured TE and TM polarized insertion loss. (b) Extracted polarization dependent loss (PDL) and excess insertion loss (EIL). (c) Calculated figure of merits (FOMs). In (a) - (c), (i) shows the results for 1.5 -cm-long uniformly coated waveguides $(0,1,2$, ..., 10 layers of GO) and (ii) shows the results for 1.5-cm-long waveguides with 2-mm-long patterned GO (0, 10, 20, ..., 100 layers of GO). (d) Polar diagrams presenting the polarizer performance for (i) 1.5-cm GO coating length, 10 layers of GO and (ii) 2-mm GO coating length, 100 layers of GO. The polar angle represents the angle between the input polarization plane and the substrate. The input $\mathrm{CW}$ power and wavelength in (a) and (d) are $0 \mathrm{dBm}$ and $1550 \mathrm{~nm}$, respectively. 
Figure 2 shows the polarization dependent (TE (in-plane) and TM (out-of-plane)) performance for both the 1.5-cm-long uniformly coated waveguides (0-10 layers), left side (i), and the patterned 2-mm-long devices (10-100 layers), right side (ii). Figure 2(a) shows the polarization dependent insertion loss. The data points depict the average values obtained from the experimental results of 3 duplicate devices and the error bars illustrate the variations for different samples. Figure 2(b) presents the PDL and EIL calculated based on the average insertion loss in Figure 2(a). Figures 2(c) and (d) show the FOMs calculated based on Figure 2(b) and the polar diagrams for the insertion loss, respectively.

The TE insertion loss increases much more strongly than TM with layer number, thus yielding a large PDL with low EIL and forming the basis for our high-performance polarization dependent devices. The PDL reached a maximum of $\sim 37.4 \mathrm{~dB}$ for a 10 layer uniformly coated device and $\sim 53.8 \mathrm{~dB}$ for a 100 layer patterned device, with a modest maximum EIL of $\sim 5.0 \mathrm{~dB}$ and $\sim 7.5 \mathrm{~dB}$ for the two devices, respectively. By optimizing the waveguide geometry to achieve a better mode overlap with the GO films, the EIL can be further reduced. Moreover, the PDL was still increasing at a rate of 2-3 $\mathrm{dB} / \mathrm{cm} /$ layer at 100 layers, and so substantially higher PDL can be obtained using layers thicker than $200 \mathrm{~nm}$. Both FOM $\mathrm{EPL}_{\mathrm{EL}}$ and $\mathrm{FOM}_{\mathrm{PDL}}$ increase with a maximum of $\mathrm{FOM}_{\mathrm{EPL}}$ and $\mathrm{FOM}_{\mathrm{PDL}}$ of $\sim 8.2$ and $\sim 8.1$, respectively, at about 50 layers with the difference between them subsequently decreasing. This is because the impact of the background PDL ( $\sim 0.3 \mathrm{~dB})$ becomes smaller as the EIL increases for increased GO layer numbers.

\section{GO-COATED POLARIZATION-SELECTIVE MRRS}

We coated GO films onto integrated MRRs to implement polarization-selective MRRs, for applications such as polarization-handling devices in coherent receivers [28]. Figure 3(a) shows a schematic of the GO-coated polarization selective MRR, with the insets showing schematic atomic structure of GO and scanning electron microscope (SEM) image of the GO film with up to 5 layers of GO. The unclad MRR made from high-index doped silica glass was fabricated via the same CMOS compatible processes as for the integrated waveguides in Section $2[29,30]$. The ring and the bus waveguide had the same waveguide geometry as in Section 2. The radius of the MRR was $\sim 592 \mu \mathrm{m}$, corresponding to a free spectral range of $\sim 0.4 \mathrm{~nm}(\sim 50 \mathrm{GHz})$. The gap between the ring and the bus waveguide was $\sim 0.8 \mu \mathrm{m}$. We used the same method to couple CW light to the MRR. We fabricated and tested two types of GO-coated MRR polarizers, uniformly coated with 1-5 layers of GO and patterned (50 $\mu \mathrm{m}$ in length) with 10-100 layers of GO using the same photolithography and lift-off processes as for the patterned waveguide in Section 2. Gold markers, prepared by metal lift-off after photolithography and electron beam evaporation, were used for precise alignment and accurate placement of GO on the MRR (deviation < $20 \mathrm{~nm}$ ). Microscope images of the integrated MRR uniformly coated with 5 layers of GO and patterned with 50 layers of GO are presented in Figures 3(b) and (c), respectively. Note that although a number of concentric rings are shown, only the center ring was coupled with the through/drop bus waveguides to form a MRR - the rest were simply used to enable easy identification by eye.
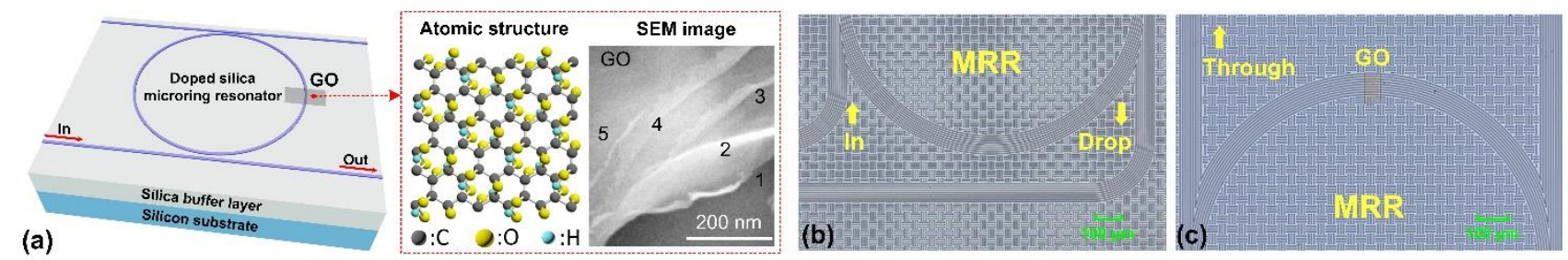

Figure 3. (a) Illustration of GO-based polarization-selective MRR. Insets show schematic atomic structure of GO and a scanning electron microscope (SEM) image of layered GO film. The numbers in the SEM refer to the number of layers for that part of the image. (b)-(c) Microscope images of the integrated MRR uniformly coated with 5 layers of GO and patterned with 50 layers of GO, respectively.

The measured TE and TM polarized transmission spectra of the uniformly GO-coated MRR are shown in Figures 4(a-i) and (a-ii), respectively, while the transmission spectra of the patterned MRR are shown in Figures 4(b-i) and (b-ii), all measured with the same doped silica MRR at a CW power of $\sim 0 \mathrm{dBm}$. The resulting $\mathrm{Q}$ factors [31-33] and extinction ratios $[34,35]$ are shown in Figure 5(a). The uncoated MRR had high extinction ratios $(>15 \mathrm{~dB})$ and relatively high $Q$ factors $(180,000)$ (although significantly less than for buried waveguides) for both polarizations. Those values decreased with GO layer number - particularly for the TE polarization, as expected. For the patterned MRR with 50 layers of GO, a maximum polarization extinction ratio (defined as the difference between the extinction ratios of the TE and TM polarized resonances) of $\sim 8.3 \mathrm{~dB}$ was achieved. This can be further improved by optimizing the waveguide geometry, GO film thickness, and coating length to properly balance the mode overlap and material anisotropy. The propagation loss of the GO hybrid integrated waveguides for TE and TM polarizations was obtained using the scattering matrix method [36-39] 
to fit the measured spectra in Figures 4(a) and 4(b) and is shown for uniformly coated ( $0-5$ layers) and patterned rings $(0-100$ layers) in Figures 5(b-i) and (b-ii), respectively, along with the waveguide propagation loss obtained from the waveguide experiment. Since different resonances did not show a significant variation over small wavelength ranges, we only fit one resonance around $1549.5 \mathrm{~nm}$ in each measured spectrum. The fit coupling coefficients between the ring and the bus waveguide for TE and TM polarizations are $\sim 0.241$ and $\sim 0.230$, respectively. The close agreement reflects the stability and reproducibility of our layer-by-layer GO film coating method. We also note that the propagation loss obtained from the ring resonator experiment is slightly higher than that obtained from the waveguide experiment, especially for the TE polarization. This probably results from photo-thermal reduction of GO in the resonant cavity at higher intensity [40].

\section{(a-i) TE}

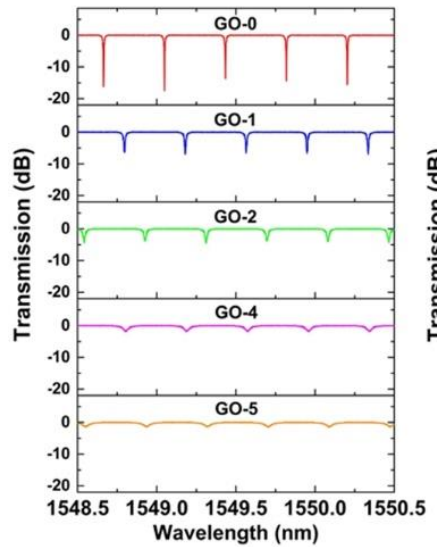

(a-ii) TM

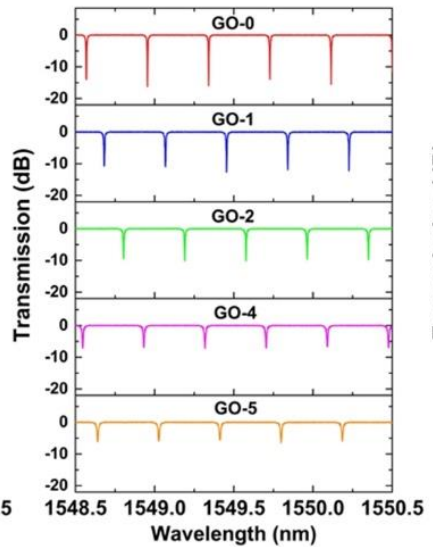

(b-i) TE

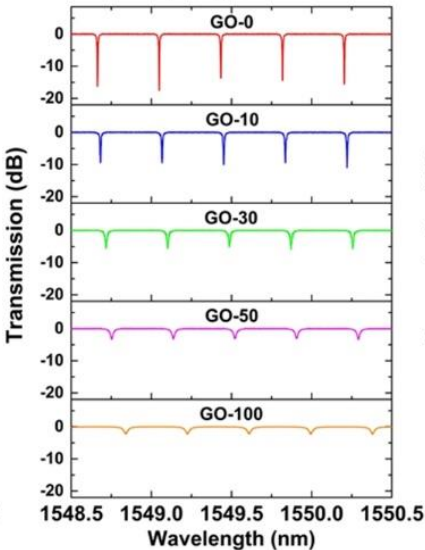

(b-ii) TM

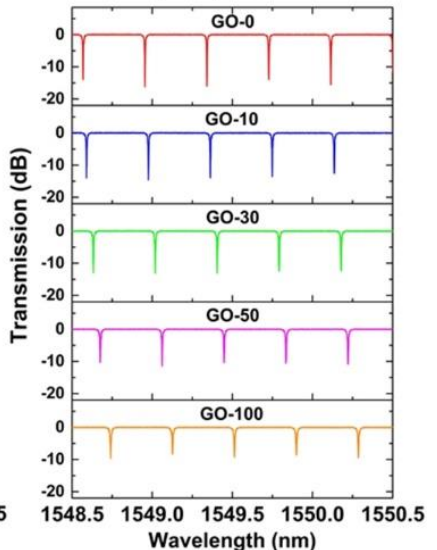

Figure 4. Measured transmission spectra of the MRR (a) uniformly coated with 0-5 layers and (d) patterned with 0-100 layers of GO.
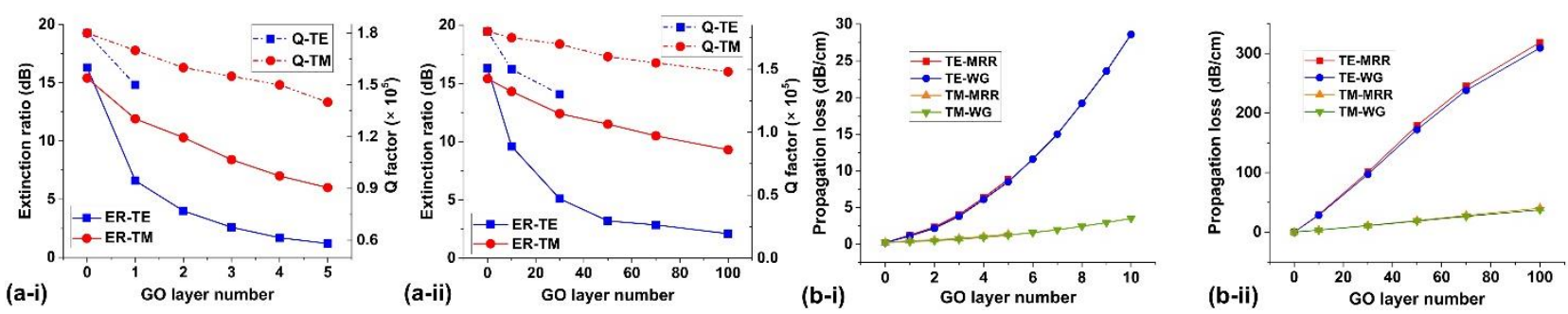

Figure 5. (a) Fit extinction ratios (ERs) and Q factors (Q) for the MRR (i) uniformly coated with 0-5 layers of GO and (ii) patterned with 0-100 layers of GO. The Q factors are not shown when the ER is $<5 \mathrm{~dB}$. (b) Fit propagation loss obtained from the MRR experiment and extracted propagation loss obtained from the waveguide experiment. (i) 0-10 layers of GO, (ii) 0-100 layers of GO.

\section{CONCLUSION}

We demonstrate waveguide polarizers and polarization-selective MRRs incorporated with layered GO films. We achieve precise control of the placement, thickness, and length of the GO films using layer-by-layer coating of GO films followed by photolithography and lift-off. We achieve a high PDL of $\sim 53.8 \mathrm{~dB}$ for the patterned GO-coated waveguide, and for the GO-coated integrated MRR an $\sim 8.3-\mathrm{dB}$ polarization extinction ratio between the TE and TM resonances. We find that the PDL is dominated by material loss anisotropy of the GO film for thin films, and by polarization dependent mode overlap for thick films. These integrated GO hybrid waveguide polarizers and polarization-selective MRRs offer a powerful new way to implement high-performance polarization selective devices for large-scale PICs.

\section{ACKNOWLEDGEMENTS}

This work was supported by the Australian Research Council Discovery Projects Programs (No. DP150102972 and DP150104327) and the Swinburne ECR-SUPRA program. We also acknowledge the Swinburne Nano Lab for the support in device fabrication and characterization. RM acknowledges support by the Natural Sciences and Engineering Research Council of Canada (NSERC) through the Strategic, Discovery and Acceleration Grants Schemes, by the MESI PSR-SIIRI Initiative in Quebec, and by the Canada Research Chair Program. 


\section{REFERENCES}

[1] D. X. Dai, J. Bauters, and J. E. Bowers, "Passive technologies for future large-scale photonic integrated circuits on silicon: polarization handling, light non-reciprocity and loss reduction," Light-Science \& Applications, vol. 1, Mar, 2012.

[2] D. X. Dai, L. Liu, S. M. Gao, D. X. Xu, and S. L. He, "Polarization management for silicon photonic integrated circuits," Laser \& Photonics Reviews, vol. 7, no. 3, pp. 303-328, May, 2013.

[3] X. Xu, J. Wu, L. Jia, M. Tan, T. G. Nguyen, S. T. Chu, B. E. Little, R. Morandotti, A. Mitchell, and D. J. Moss, “Continuously tunable orthogonally polarized RF optical single sideband generator based on micro-ring resonators," Journal of Optics, vol. 20, no. 11, pp. 115701, 2018.

[4] X. Xu, J. Wu, M. Tan, T. G. Nguyen, S. Chu, B. Little, R. Morandotti, A. Mitchell, and D. J. Moss, "Orthogonally polarized RF optical single sideband generation and dual-channel equalization based on an integrated micro-ring resonator," Journal of Lightwave Technology, vol. 36, no. 20, pp4808-4818, 2018.

[5] Y. Zhang, Y. He, J. Wu, X. Jiang, R. Liu, C. Qiu, X. Jiang, J. Yang, C. Tremblay, and Y. Su, "High-extinction-ratio silicon polarization beam splitter with tolerance to waveguide width and coupling length variations," Opt Express, vol. 24, no. 6, pp. 6586-93, Mar 21, 2016.

[6] L. Lu, J. Wu, T. Wang, and Y. Su, "Compact all-optical differential-equation solver based on silicon microring resonator," Frontiers of Optoelectronics, vol. 5, no. 1, pp. 99-106, 2012.

[7] L. Zhang, J. Wu, X. Yin, X. Sun, P. Cao, X. Jiang, and Y. Su, “A High-Speed Second-Order Photonic Differentiator Based on Two-Stage Silicon Self-Coupled Optical Waveguide," IEEE Photonics Journal, vol. 6, no. 2, pp. 1-5, 2014.

[8] J. Wu, P. Cao, X. Hu, X. Jiang, T. Pan, Y. Yang, C. Qiu, C. Tremblay, and Y. Su, "Compact tunable silicon photonic differential-equation solver for general linear time-invariant systems," Opt Express, vol. 22, no. 21, pp. 26254-64, Oct 20, 2014.

[9] J. Wu, P. Cao, T. Pan, Y. Yang, C. Qiu, C. Tremblay, and Y. Su, "Compact on-chip $1 \times 2$ wavelength selective switch based on silicon microring resonator with nested pairs of subrings," Photonics Research, vol. 3, no. 1, pp. 9, 2014.

[10] S. Lai, Z. Xu, B. Liu, and J. Wu, "Compact silicon photonic interleaver based on a self-coupled optical waveguide," Appl Opt, vol. 55, no. 27, pp. 7550-5, Sep 20, 2016.

[11] J. Wu, B. Liu, J. Peng, J. Mao, X. Jiang, C. Qiu, C. Tremblay, and Y. Su, "On-Chip Tunable Second-Order DifferentialEquation Solver Based on a Silicon Photonic Mode-Split Microresonator," Journal of Lightwave Technology, vol. 33, no. 17, pp. 3542-3549, 2015.

[12] L. Lu, F. Li, M. Xu, T. Wang, J. Wu, L. Zhou, and Y. Su, "Mode-Selective Hybrid Plasmonic Bragg Grating Reflector," IEEE Photonics Technology Letters, vol. 24, no. 19, pp. 1765-1767, 2012.

[13] Q. Bao, H. Zhang, B. Wang, Z. Ni, C. H. Y. X. Lim, Y. Wang, D. Y. Tang, and K. P. Loh, "Broadband graphene polarizer," Nature Photonics, vol. 5, no. 7, pp. 411-415, 2011.

[14] J. T. Kim, and C. G. Choi, "Graphene-based polymer waveguide polarizer," Optics Express, vol. 20, no. 4, pp. 3556-3562, Feb 13, 2012.

[15] W. H. Lim, Y. K. Yap, W. Y. Chong, C. H. Pua, N. M. Huang, R. M. De La Rue, and H. Ahmad, "Graphene oxide-based waveguide polariser: from thin film to quasi-bulk," Opt Express, vol. 22, no. 9, pp. 11090-8, May 5, 2014.

[16] H. Lin, Y. Song, Y. Huang, D. Kita, S. Deckoff-Jones, K. Wang, L. Li, J. Li, H. Zheng, Z. Luo, H. Wang, S. Novak, A. Yadav, C.-C. Huang, R.-J. Shiue, D. Englund, T. Gu, D. Hewak, K. Richardson, J. Kong, and J. Hu, "Chalcogenide glass-on-graphene photonics," Nature Photonics, vol. 11, no. 12, pp. 798-805, 2017.

[17] Y. Yang, R. Liu, J. Wu, X. Jiang, P. Cao, X. Hu, T. Pan, C. Qiu, J. Yang, Y. Song, D. Wu, and Y. Su, "Bottom-up Fabrication of Graphene on Silicon/Silica Substrate via a Facile Soft-hard Template Approach,” Sci Rep, vol. 5, pp. 13480, Aug $27,2015$.

[18] X. Sun, C. Qiu, J. Wu, H. Zhou, T. Pan, J. Mao, X. Yin, R. Liu, W. Gao, Z. Fang, and Y. Su, "Broadband photodetection in a microfiber-graphene device," Opt Express, vol. 23, no. 19, pp. 25209-16, Sep 21, 2015.

[19] Y. Yang, J. Wu, X. Xu, Y. Liang, S. T. Chu, B. E. Little, R. Morandotti, B. Jia, and D. J. Moss, "Invited Article: Enhanced four-wave mixing in waveguides integrated with graphene oxide," APL Photonics, vol. 3, no. 12, pp. 120803, 2018.

[20] J. Wu, Y. Yang, Y. Qu, X. Xu, Y. Liang, S. T. Chu, B. E. Little, R. Morandotti, B. Jia, and D. J. Moss, “Graphene Oxide Waveguide and Micro-Ring Resonator Polarizers," Laser \& Photonics Reviews, vol. 13, no. 9, 1900056 Sep, 2019.

[21] X. Li, H. Ren, X. Chen, J. Liu, Q. Li, C. Li, G. Xue, J. Jia, L. Cao, A. Sahu, B. Hu, Y. Wang, G. Jin, and M. Gu, “Athermally photoreduced graphene oxides for three-dimensional holographic images," Nat Commun, vol. 6, pp. 6984, Apr 22, 2015.

[22] X. Zheng, B. Jia, H. Lin, L. Qiu, D. Li, and M. Gu, "Highly efficient and ultra-broadband graphene oxide ultrathin lenses with three-dimensional subwavelength focusing," Nat Commun, vol. 6, pp. 8433, Sep 22, 2015.

[23] X. Xu, J. Wu, M. Shoeiby, T. G. Nguyen, S. T. Chu, B. E. Little, R. Morandotti, A. Mitchell, and D. J. Moss, "Reconfigurable broadband microwave photonic intensity differentiator based on an integrated optical frequency comb source," $A P L$ Photonics, vol. 2, no. 9, pp. 096104, 2017.

[24] X. Xu, J. Wu, T. G. Nguyen, S. T. Chu, B. E. Little, R. Morandotti, A. Mitchell, and D. J. Moss, "Broadband RF Channelizer Based on an Integrated Optical Frequency Kerr Comb Source," Journal of Lightwave Technology, vol. 36, no. 19, pp. 45194526, 2018.

[25] J. Wu, X. Xu, T. G. Nguyen, S. T. Chu, B. E. Little, R. Morandotti, A. Mitchell, and D. J. Moss, "RF Photonics: An Optical Microcombs' Perspective,” IEEE Journal of Selected Topics in Quantum Electronics, vol. 24, no. 4, pp. 1-20, 2018. 
[26] X. Xu, J. Wu, T. G. Nguyen, T. Moein, S. T. Chu, B. E. Little, R. Morandotti, A. Mitchell, and D. J. Moss, "Photonic microwave true time delays for phased array antennas using a 49 GHz FSR integrated optical micro-comb source [Invited]," Photonics Research, vol. 6, no. 5, pp. B30, 2018.

[27] X. Xu, J. Wu, T. G. Nguyen, M. Shoeiby, S. T. Chu, B. E. Little, R. Morandotti, A. Mitchell, and D. J. Moss, “Advanced RF and microwave functions based on an integrated optical frequency comb source," Opt Express, vol. 26, no. 3, pp. 2569-2583, Feb 5, 2018.

[28] Y. Tan, S. Chen, and D. Dai, "Polarization-selective microring resonators," Opt Express, vol. 25, no. 4, pp. 4106-4119, Feb 20, 2017.

[29] X. Xu, M. Tan, J. Wu, T. G. Nguyen, S. Chu, B. Little, R. Morandotti, A. Mitchell, and D. J. Moss, "Advanced adaptive photonic RF filters with 80 taps based on an integrated optical micro-comb source," Journal of Lightwave Technology, vol.37, no. 4, pp1288-1295, 2019.

[30] X. Xu, M. Tan, J. Wu, T. G. Nguyen, S. T. Chu, B. E. Little, R. Morandotti, A. Mitchell, and D. J. Moss, "High performance RF filters via bandwidth scaling with Kerr micro-combs," APL Photonics, vol. 4, no. 2, pp. 026102, 2019.

[31] J. Wu, T. Moein, X. Xu, G. Ren, A. Mitchell, and D. J. Moss, "Micro-ring resonator quality factor enhancement via an integrated Fabry-Perot cavity," APL Photonics, vol. 2, no. 5, pp. 056103, 2017.

[32] J. Wu, T. Moein, X. Xu, and D. J. Moss, "Advanced photonic filters based on cascaded Sagnac loop reflector resonators in silicon-on-insulator nanowires," APL Photonics, vol. 3, no. 4, pp. 046102, 2018.

[33] T. Pan, C. Qiu, J. Wu, X. Jiang, B. Liu, Y. Yang, H. Zhou, R. Soref, and Y. Su, “Analysis of an electro-optic modulator based on a graphene-silicon hybrid 1D photonic crystal nanobeam cavity," Opt Express, vol. 23, no. 18, pp. 23357-64, Sep 7, 2015.

[34] T. Wang, M. Xu, F. Li, J. Y. Wu, L. J. Zhou, and Y. K. Su, "Design of a high-modulation-depth, low-energy silicon modulator based on coupling tuning in a resonance-split microring," Journal of the Optical Society of America B-Optical Physics, vol. 29, no. 11, pp. 3047-3056, Nov, 2012.

[35] X. Mu, W. Jiayang, W. Tao, H. Xiaofeng, J. Xinhong, and S. Yikai, "Push-Pull Optical Nonreciprocal Transmission in Cascaded Silicon Microring Resonators," IEEE Photonics Journal, vol. 5, no. 1, pp. 2200307-2200307, 2013.

[36] X. Jiang, J. Wu, Y. Yang, T. Pan, J. Mao, B. Liu, R. Liu, Y. Zhang, C. Qiu, C. Tremblay, and Y. Su, "Wavelength and bandwidth-tunable silicon comb filter based on Sagnac loop mirrors with Mach-Zehnder interferometer couplers," Opt Express, vol. 24, no. 3, pp. 2183-8, Feb 8, 2016.

[37] J. Wu, P. Cao, X. Hu, T. Wang, M. Xu, X. Jiang, F. Li, L. Zhou, and Y. Su, "Nested Configuration of Silicon Microring Resonator With Multiple Coupling Regimes," IEEE Photonics Technology Letters, vol. 25, no. 6, pp. 580-583, 2013.

[38] J. Wu, X. Jiang, T. Pan, P. Cao, L. Zhang, X. Hu, and Y. Su, "Non-blocking $2 \times 2$ switching unit based on nested silicon microring resonators with high extinction ratios and low crosstalks," Chinese Science Bulletin, vol. 59, no. 22, pp. 2702-2708, 2014.

[39] J. Wu, J. Peng, B. Liu, T. Pan, H. Zhou, J. Mao, Y. Yang, C. Qiu, and Y. Su, "Passive silicon photonic devices for microwave photonic signal processing," Optics Communications, vol. 373, pp. 44-52, 2016.

[40] W. Y. Chong, W. H. Lim, Y. K. Yap, C. K. Lai, R. M. De La Rue, and H. Ahmad, "Photo-induced reduction of graphene oxide coating on optical waveguide and consequent optical intermodulation," Sci Rep, vol. 6, pp. 23813, Apr 1, 2016. 